\title{
Intrinsic ambipolarity and rotation in stellarators
}

\author{
P. Helander ${ }^{1}$ and A. N. Simakov ${ }^{2}$ \\ ${ }^{1}$ Max-Planck-Institut für Plasmaphysik, 17491 Greifswald, Germany \\ ${ }^{2}$ Los Alamos National Laboratory, Los Alamos, NM 87544, USA
}

(Dated: May 27, 2008)

\begin{abstract}
It is demonstrated that collisional plasma transport is intrinsically ambipolar only in magnetic configurations that are quasiaxisymmetric or quasihelically symmetric. Only in such configurations can the plasma rotate freely, and then only in the direction of quasisymmetry. In a nonquasisymmetric magnetic field, it is shown that the average radial electric field is determined by parallel viscosity, which in turn is usually governed by collisional processes. Locally, the radial electric field may be affected by turbulent Reynolds stress producing zonal flows, but on a radial average, taken on a length scale exceeding the ion gyroradius, it is determined by parallel viscosity, at least if the turbulence is electrostatic and obeys the conventional gyrokinetic orderings. This is different from the situation in a tokamak or a quasisymmetric stellarator, where there is no flow damping by parallel viscosity in the symmetry direction and the turbulent Reynolds stress may affect the global radial electric field.
\end{abstract}


An important characteristic of a magnetically confined plasma is whether it is able to rotate. In this Letter we establish in which magnetic configurations it can. It is well known that an axisymmetric tokamak plasma can rotate (almost) freely in the toroidal direction, but poloidal rotation turns out to be inhibited. This is because poloidal rotation is damped by ion collsions through parallel viscosity, on the collision time scale, while toroidal rotation is damped only by cross-field viscosity. The latter can be caused by either collisions or turbulence but operates on the confinement time scale. The confinement time for angular momentum is comparable to that of energy and is typically at least two orders of magnitude longer than the collision time. The fact that collisions do not lead to rapid damping of toroidal rotation is expressed by the statement that "neoclassical transport is instrinsically ambipolar" $[1,2]$. This means that the radial current vanishes in leading order (in a gyroradius expansion) and is independent of the radial electric field. The latter can therefore assume any value, which means that the plasma can rotate at any speed [3].

It is not obvious a priori what the corresponding situation should be in a stellarator. Great effort has gone into optimizing stellarator magnetic configurations to minimize the collisonal "neoclassical" transport, which otherwise tends to be prohibitively large. Two main optimization strategies have emerged based on the concepts of quasisymmetry and omnigeneity, respectively. The more conservative approach is to make the magnetic field quasiaxisymmetric [4] or quasihelically symmetric [5], which means that the magnetic field strength does not depend on both Boozer angles but only on a linear combination thereof $[6,7]$. In a perfectly quasisymmetric magnetic field all neoclassical properties are identical (in leading order) to those in the tokamak [8]. A plasma confined by such a field therefore enjoys instrinsic ambipolarity and is free to rotate in the direction of symmetry $[9,10]$. In practice it is not possible to create a perfectly quasisymmetric magnetic field, but it is possible to come close. Currently the most quasisymmetric stellarator is the HSX experiment [11], where the "effective helical ripple" is less than $1 \%$ over most of the plasma volume. Quasisymmetry is a special case of omnigeneity, which means that there is no net (timeaveraged) drift of particles off flux surfaces [12-14]. Omnigenous configurations therefore have low neoclassical transport, but it is not immediately clear whether they can support plasma rotation. Here we show that the only magnetic configurations where instrinsic ambipolarity holds are the quasisymmetric ones. Even in a perfectly omnigenous magnetic field, the cross-field transport rates of ions and electrons are unequal unless the electric field 
is fixed at a certain value. The rotation is thus clamped at the corresponding speed, at least on time scales longer than the ion collision time. In fact, both its direction and magnitude are fixed, and we argue that this state of affairs is unlikely to be affected by turbulence. As shown below, on a radial average taken over many gyroradii, the Reynolds stress from standard (electrostatic) gyrokinetic turbulence is too weak to significantly influence the radial electric field. Only if the level of turbulent transport is much higher than implied by the standard gyrokinetic orderings is the Reynolds stress of significance, unless the magnetic field is quasisymmetric. On the other hand, in a quasisymmetric field no amount of turbulence can produce parallel viscous damping of plasma rotation in leading order.

We begin the analysis by considering collisional transport in a plasma without turbulence. The magnetic field is arbitrary but is assumed to possess nested flux surfaces labeled by $\psi$. As usual in the theory of plasma confinement, we assume that the gyroradius is shorter than the macroscopic scale length, and expand in the corresponding small parameter, $\delta=\rho / L \ll 1$. In lowest order the distribution function of each species $a$ is then Maxwellian whose pressure $p_{a 0}$ and temperature $T_{a 0}$ are constant on flux surfaces. In next order, the gyro-averaged part of the distribution function is determined from the drift kinetic equation

$$
v_{\|} \nabla_{\|} \bar{f}_{a 1}+\mathbf{v}_{d} \cdot \nabla f_{a 0}=C_{a}\left(\bar{f}_{a 1}\right)
$$

where the independent variables are the energy $E=m_{a} v^{2} / 2+e_{a} \phi$ and magnetic moment $\mu=m_{a} v_{\perp}^{2} / 2 B$. The drift velocity $\mathbf{v}_{d}$ comprises the sum of the magnetic and $E \times B$ drifts. The contribution from latter vanishes in lowest order since the electrostatic potential is approximately constant on flux surfaces, $\phi \simeq \phi_{0}(\psi)$, but there is a first-order drift $\mathbf{B} \times$ $\nabla \phi_{1} / B^{2}$. Multiplying the kinetic equation by $\bar{f}_{a 1} / f_{a 0}$, integrating over velocity space and taking a flux-surface average $\langle\cdots\rangle$ gives an entropy balance equation,

$$
\left\langle\boldsymbol{\Gamma}_{a} \cdot\left(\frac{\nabla p_{a 0}}{p_{a 0}}+\frac{e_{a} \nabla \phi_{0}}{T_{a 0}}\right)+\frac{\mathbf{q}_{a} \cdot \nabla T_{a 0}}{T_{a 0}^{2}}\right\rangle=\left\langle\int d^{3} v \bar{f}_{a 1} C_{a}\left(\bar{f}_{a 1}\right) / f_{a 0}\right\rangle
$$

where $\boldsymbol{\Gamma}_{a}$ and $\mathbf{q}_{a}$ are the particle and heat fluxes carried by $\bar{f}_{a 1}$. Since Eq. (1) is linear, these are linear combinations of the radial pressure, electrostatic potential and temperature gradients. Their contributions can thus be treated separately, and we consider the case without pressure or temperature gradients. For simplicity, we also choose the radial electric field to be so small that the poloidal $E \times B$ drift can be neglected. Multiplying by $T_{a 0}$ and 
summing over all species gives an expression for the radial current,

$$
\phi_{0}^{\prime}(\psi)\langle\mathbf{j} \cdot \nabla \psi\rangle=\sum_{a} T_{a 0}\left\langle\int d^{3} v \bar{f}_{a 1} C_{a}\left(\bar{f}_{a 1}\right) / f_{a 0}\right\rangle \leq 0 .
$$

By definition, the transport is intrinsically ambipolar if, and only if, this current vanishes, regardless of the value of $\phi_{0}^{\prime}(\psi)$ (as long as it is small enough to satisfy the linear approximation). The H-theorem then implies that $\bar{f}_{a 1}$ is of the form [15]

$$
\bar{f}_{a 1}=\left(\alpha_{a}+\beta_{a} v_{\|}+\gamma_{a} v^{2}\right) f_{a 0}
$$

where $\alpha_{a}, \beta_{a}$ and $\gamma_{a}$ may depend on position but not on velocity. Moreover, the flow velocities of all species must be equal, $T_{a 0} \beta_{a} / m_{a}=T_{b 0} \beta_{b} / m_{b}$, and strictly speaking the temperaures must also be equal, $T_{a 0}=T_{b 0}$. However, for particle species with very disparate masses, such as electrons and ions, the energy exchange is very small, and if it is neglected in the corresponding collision operators then the H-theorem does not imply equal temperatures. In any case, since the collision operator vanishes whenever the current (2) does, it follows from the kinetic equation (1) that the part of $\bar{f}_{a 1}$ that is caused by the radial electric field is odd in $v_{\|}$, so that $\alpha_{a}$ and $\gamma_{a}$ vanish. But then

$$
v_{\|} \nabla_{\|} \bar{f}_{a 1}=v^{2}\left[(1-\lambda B) \nabla_{\|} \beta_{a}-\beta_{a} \lambda \nabla_{\|} B / 2\right] f_{a 0}
$$

must equal

$$
-\mathbf{v}_{d} \cdot \nabla f_{a 0}=\frac{m_{a} v^{2}}{e_{a} B^{3}} \frac{\partial f_{a 0}}{\partial \psi}\left(1-\frac{\lambda B}{2}\right)(\mathbf{B} \times \nabla B) \cdot \nabla \psi,
$$

for all values of $\lambda=2 \mu / m_{a} v^{2}$. (The radial $E \times B$ drift has been neglected here, being smaller than the corresponding magnetic drift in the gyroradius expantion.) This requires

$$
\frac{m_{a}}{e B^{2}} \frac{\partial f_{a 0}}{\partial \psi}(\mathbf{B} \times \nabla \psi) \cdot \nabla B=B \nabla_{\|} \beta_{a}=2 B \nabla_{\|} \beta_{a}+\beta_{a} \nabla_{\|} B
$$

and thus implies

$$
(\mathbf{B} \times \nabla \psi) \cdot \nabla \ln B=F(\psi) \nabla_{\|} B
$$

for some flux function $F(\psi)$. Only when this condition is satisfied is the transport intrinsically ambipolar.

To find the relation of this result to quasisymmetry, we represent the magnetic field in Boozer coordinates $(\psi, \theta, \varphi)$,

$$
\mathbf{B}=\beta(\psi, \theta, \varphi) \nabla \psi+I(\psi) \nabla \theta+J(\psi) \nabla \varphi=\nabla \psi \times \nabla(\theta-\iota \varphi)
$$


where $\iota(\psi)$ denotes the rotational transform, and $I(\psi)$ and $J(\psi)$ are the toroidal and poloidal currents, respectively. Equation (3) now becomes

$$
J \frac{\partial B}{\partial \theta}-I \frac{\partial B}{\partial \varphi}=F(\psi)\left(\iota \frac{\partial B}{\partial \theta}+\frac{\partial B}{\partial \varphi}\right) .
$$

If $B$ is Fourier transformed,

$$
B(\psi, \theta, \varphi)=\sum_{m, n} \alpha_{m, n}(\psi) e^{i(m \theta-n \varphi)}
$$

this requires that the condition

$$
[m J+n I-F(m \iota-n)] \alpha_{m, n}=0
$$

should be satisfied for all $(m, n)$. This can only hold if $\alpha_{m, n}=0$ or

$$
F(\psi)=\frac{(m / n) J(\psi)+I(\psi)}{(m / n) \iota(\psi)-1} .
$$

Since the left-hand side is independent of $m / n$, this relation can only be satisfied for one particular value of this ratio, $M / N$ say. The magnetic field variation over the flux surface then only contains the corresponding helicity

$$
B=\sum_{k} \alpha_{k M, k N}(\psi) e^{i k(M \theta-N \varphi)},
$$

and thus can be written as $B=B(\psi, M \theta-N \varphi)$. It follows, in other words, that the magnetic field in an intrinsically ambipolar configuration must necessarily be quasisymmetric. By adding the $E \times B$ velocity to the parallel flow velocity carried by $\bar{f}_{a 1}$ it is straighforward to show that the plasma rotates in the symmetry direction (when the temperature gradient vanishes), as one would expect.

In order to assess the robustness of this result, we now consider the effect of electrostatic turbulence on the radial electric field. The magnetic field is assumed to be general, i.e., not necessarily quasisymmetric. We start by constructing a vector field

$$
\mathbf{G}=\frac{\mathbf{b} \times \nabla \psi}{B}+u \mathbf{B}
$$

where $\mathbf{b}=\mathbf{B} / B$ and the function $u(\psi, \theta, \varphi)$ is chosen so that

$$
\mathbf{b} \cdot \nabla u=\frac{2}{B^{2}}(\mathbf{b} \times \nabla \psi) \cdot \nabla \ln B
$$


which implies $\nabla \cdot \mathbf{G}=0$. The integrability condition for this equation is satisfied if the equilibrium has isotropic pressure in lowest order, i.e., $\mathbf{j} \times \mathbf{B}=\nabla p_{0}$. In a quasisymmetric field $u=-F(\psi) / B^{2}$. We now take the scalar product of $\mathbf{G}$ with the momentum equation

$$
\frac{\partial(\rho \mathbf{V})}{\partial t}+\nabla \cdot(\rho \mathbf{V} \mathbf{V}+p \mathbf{I}+\boldsymbol{\pi})=\mathbf{j} \times \mathbf{B}
$$

where $\rho$ is the plasma density, $\mathbf{V}$ the flow velocity, $\boldsymbol{\pi}$ the viscosity, and $\mathbf{I}$ the identity tensor. On an average over time and over a flux surface we obtain

$$
\begin{gathered}
\langle\mathbf{j} \cdot \nabla \psi\rangle=-\langle\nabla \cdot(\rho \mathbf{V} \mathbf{V}+p \mathbf{I}+\boldsymbol{\pi}) \cdot \mathbf{G}\rangle \\
=\langle(\rho \mathbf{V} \mathbf{V}+\boldsymbol{\pi}): \nabla \mathbf{G}\rangle-\frac{1}{V^{\prime}(\psi)} \frac{\partial}{\partial \psi}\left\langle V^{\prime}(\psi)(\rho \mathbf{V V}+\boldsymbol{\pi}): \mathbf{G} \nabla \psi\right\rangle,
\end{gathered}
$$

where $V(\psi)$ is the volume within the flux surface $\psi$. This expression for the average radial current in terms of viscosity $\boldsymbol{\pi}$ and Reynolds stress $\rho \mathbf{V V}$ is quite general. However, interesting conclusions can be drawn if we assume that any turbulent fluctuations obey the orderings usually made in gyrokinetic theory. The viscosity is in lowest order given by the parallel viscosity tensor

$$
\boldsymbol{\pi}=\left(p_{\|}-p_{\perp}\right)(\mathbf{b b}-\mathbf{I} / 3)+O\left(\delta^{2} p\right)
$$

where the pressure anisotropy is of order $\left(p_{\|}-p_{\perp}\right) / p \sim \delta$, and the flow velocity is usually assumed to be of order

$$
\mathbf{V} \sim \delta v_{T}
$$

where $v_{T}$ is the thermal ion speed. It follows that the Reynolds stress in Eq. (4) is smaller than the viscosity, $\rho \mathbf{V V} \ll \boldsymbol{\pi}$. However, since fluctuating quantities in gyrokinetics may vary on the the gyroradius length scale $\rho$ while equilibrium quantities only vary on the macroscopic scale length $L$, the Reynolds stress and fluctuating portions of viscosity in the last term of Eq. (4) are not necessarily negligible. On the other hand, if we take an integral over the volume between two flux surfaces, $\psi_{1}$ and $\psi_{2}$, so as to get an expression for the volume averaged radial current,

$$
\int_{\psi_{1}}^{\psi_{2}}\langle\mathbf{j} \cdot \nabla \psi\rangle V^{\prime} d \psi=\int_{\psi_{1}}^{\psi_{2}}\langle\boldsymbol{\pi}: \nabla \mathbf{G}\rangle V^{\prime} d \psi-\left[\left\langle(\rho \mathbf{V} \mathbf{V}+\boldsymbol{\pi}): \mathbf{G} \nabla \psi V^{\prime}\right\rangle\right]_{\psi_{1}}^{\psi_{2}},
$$

then the second term on the right becomes smaller than the first one if the separation between the flux surfaces is much larger than the gyroradius. This is because if $\Delta r \sim\left(\psi_{2}-\psi_{1}\right) /|\nabla \psi|$ 
is the characteristic distance between the flux surfaces and $\Delta V \sim V^{\prime}|\nabla \psi| \Delta r$ the volume between them, then

$$
\int_{\psi_{1}}^{\psi_{2}} \pi: \nabla \mathbf{G} V^{\prime} d \psi \sim \delta p \Delta V
$$

is much larger than

$$
\left[\left\langle(\rho \mathbf{V V}+\boldsymbol{\pi}): \mathbf{G} \nabla \psi V^{\prime}\right\rangle\right]_{\psi_{1}}^{\psi_{2}} \sim \delta^{2} p \Delta V(L / \Delta r)
$$

if $\Delta r \gg \delta L$. In other words, on a volume average taken over a radial length scale exceeding the gyroradius (but possibly still smaller than the macroscopic length), the radial current is determined by parallel viscosity alone. Insofar as the parallel viscosity is determined primarily by neoclassical processes, we thus expect the average radial electric field to be set by the condition of ambipolar collisional transport. Small-scale "zonal flows" may be produced by Reynolds stress, but the average radial electric current is not. The point is that, in a tokamak or a quasisymmetric stellarator, the radial current produced by neoclassical tranport is comparable to that driven by the Reynolds stress (in terms of the $\delta$-ordering), but in a non-quasisymmetric stellarator the neoclassical current is a factor $\delta^{-1}$ larger and therefore dominates.

Before closing, it is appropriate to discuss two issues. The first one is whether the parallel viscosity is indeed set by collisional processes or turbulence. In principle it is possible that strong turbulent fluctuations may affect the first-order distribution function $\bar{f}_{1}$ to such an extent that the parallel viscosity departs from its neoclassical value. This is however difficult to achieve within the gyrokinetic ordering, where it can be shown that the collision frequency must be very small, of order $\delta^{2} v_{T} / L$, in order not to exceed the rate at which turbulent fluctuations scatter particles in velocity space through the fluctuating electric field. Furthermore, if turbulence did affect the parallel viscosity, it would also change the bootstrap current from its neoclassical value, contradicting observations both in tokamaks and stellarators.

A second point of interest is what happens to the prediction (6) in the limit of a quasisymmetric magnetic field. The parallel viscosity tensor (5) satisfies

$$
\nabla \cdot \boldsymbol{\pi}=-\frac{1}{3} \nabla \pi_{\|}+\mathbf{b} \nabla_{\|} \pi_{\|}+\pi_{\|}\left(\boldsymbol{\kappa}-\mathbf{b} \nabla_{\|} \ln B\right),
$$

where $\pi_{\|}=p_{\|}-p_{\perp}$ and

$$
\boldsymbol{\kappa}=\mathbf{b} \cdot \nabla \mathbf{b}=\nabla_{\perp} \ln B+\mu_{0} \nabla p_{0} / B^{2}
$$


is the curvature vector. Therefore

$$
\left\langle\frac{(\nabla \cdot \boldsymbol{\pi}) \cdot(\mathbf{B} \times \nabla \psi)}{B^{2}}\right\rangle=\left\langle\frac{\pi_{\|}}{3 B^{2}}(\mathbf{B} \times \nabla \psi) \cdot \nabla \ln B\right\rangle
$$

and

$$
\left\langle\frac{(\nabla \cdot \boldsymbol{\pi}) \cdot \mathbf{B}}{B^{2}}\right\rangle=\left\langle\frac{\pi_{\|} \nabla_{\|} \ln B}{3 B}\right\rangle,
$$

and it follows that $\langle(\nabla \cdot \boldsymbol{\pi}) \cdot \mathbf{G}\rangle=0$ if the quasisymmetry condition (3) holds. This means that parallel viscosity does not contribute to the radial current (4) if the magnetic field is quasisymmetric. In a non-symmetric device it is the dominant term, as we have seen, whereas in a quasisymmetric field the current is instead determined by Reynolds stress and offdiagonal components of the viscosity tensor. This reflects the situation in a tokamak, where the radial electric field is set by turbulent and neoclassical transport of angular momentum.

As already mentioned, it is not possible to achieve exact quasisymmetry. It is therefore natural to ask how nearly quasisymmetric a stellarator must be in order for it to behave like a tokamak in terms of intrinsic ambipolarity. The answer depends on the collisionality regime, since the non-ambipolar current is sensitive to collisionality. In the low-collisionality " $1 / \nu$-regime", the diffusion coefficient scales as

$$
D_{a} \sim \epsilon_{h}^{3 / 2} \delta^{2} \frac{T_{a}}{m_{a} \nu_{a}}
$$

where $\epsilon_{h}$ is the effective helical ripple and $\nu_{a}$ the collisionality. The nonambipolar current is thus of order

$$
\langle\mathbf{j} \cdot \nabla \psi\rangle \sim \epsilon_{h}^{3 / 2} \delta^{2} \frac{\Omega_{a}}{\nu_{a}},
$$

where $\Omega_{a}$ is the gyroradius. This current exceeds that driven by the Reynolds stress on a volume average, Eq. (7), if

$$
\epsilon_{h}>\left(\frac{\nu_{a} L}{\Omega_{a} \Delta r}\right)^{2 / 3}
$$

In other words, if the average is taken over the volume between two flux surfaces a distance $\Delta r=N \rho_{a}$ apart, with $N>1$, then

$$
\epsilon_{h}>\left(\frac{L}{N \lambda}\right)^{2 / 3}
$$

If the mean-free path $\lambda=v_{T a} / \nu_{a}$ is long, it is difficult to achieve intrinsic ambipolarity in practice. 
In summary, we have shown that a stellarator plasma can rotate freely only if the magnetic field is quasisymmetric. Otherwise the radial electric field is set by the condition that the collisional transport be ambipolar, and the plasma rotation is clamped at the corresponding value, at least in the absence of strong momentum sources. On a radial average, this conclusion also holds in the presence of electrostatic turbulence, as long as the usual gyrokinetic orderings are satisfied. Locally, turbulent Reynolds stress can however produce zonal flows, which therefore deserve study, particularly as they may affect the turbulent transport $[16,17]$.

We are grateful to Craig Beidler, Henning Maaßberg and Jürgen Nührenberg for helpful comments.

[1] P.H. Rutherford, Phys. Fluids 13, 482 (1970).

[2] F.L. Hinton and R.D. Hazeltine, Rev. Mod. Phys 48, 239 (1976).

[3] In the following, we therefore use the phrase "can rotate freely" as a synonom to "is intrinsically ambipolar".

[4] J. Nührenberg, W. Lotz, S. Gori, in Theory of Fusion Plasmas (Editrice Compositori, Bologna, 1994), p. 3.

[5] J. Nührenberg and R. Zille, Phys. Lett. A 129, 113 (1988).

[6] M.Yu. Isaev, M.I. Mikhailov, and V.D. Shafranov, Plasma Phys. Rep. 20, 319 (1994).

[7] A.H. Boozer, Plasma Phys. Control. Fusion 37, A103 (1995).

[8] A.H. Boozer, Phys. Fluids 26, 496 (1983).

[9] H. Sugama and S. Nishimura, Phys. Plasmas 9, 4637 (2002).

[10] D.A. Spong, Phys. Plasmas 12, 056114 (2005).

[11] J.M. Canik, D.T. Anderson, F.S. Anderson, K.M. Likin, J.N. Talmadge, and K.Zhai, Phys. Rev. Lett. 98, 085002 (2007).

[12] L.S. Hall and B. McNamara, Phys. Fluids 18, 552 (1975).

[13] J.R. Cary and S. Shasharina, Phys. Plasmas 4, 3323 (1997).

[14] It was demonstrated in Ref. [13] that an analytical magnetic field that is strictly omnigenous must also be quasisymmetric. However, this appears irrelevant in practice since it was also shown that (in principle at least) it is possible to construct fields which are very nearly (perhaps 
arbitrarily close to) omnigenous whilst being far from quasisymmetric.

[15] P. Helander and D.J. Sigmar, Collisional transport in magnetized plasmas (Cambridge University Press, 2002).

[16] H. Sugama and T.-H. Watanabe Phys. Rev. Lett. 94, 115001 (2005).

[17] P. Xanthopoulos, F. Merz, T. Görler, and F. Jenko, Phys. Rev. Lett. 99, 035002 (2007). 